\title{
Advanced Sacral Chordoma: A Multidisciplinary Team Approach
}

Kow Ren Yi ${ }^{1}$, Saiful Azlan Kamisan ${ }^{1}$, Goh Kian Liang ${ }^{1}$, Asmah Hanim Hamdan², Ahmad Razali Md Ralib@Md Raghib ${ }^{3}$

${ }^{1}$ Department of Orthopaedic Surgery, International Islamic University

${ }^{2}$ Department of Pathology, International Islamic University Malaysia

${ }^{3}$ Department of Radiology, International Islamic University Malaysia

Introduction: Sacral chordomas are rare, low-grade and slow growing malignant bone tumours arising from the sacral bone. They are locally aggressive with the tendency to metastasize to the lungs. Surgical resection remains the mainstay of treatment of sacral chordomas. However, most patients presented late with large tumours and intra-abdominal extension making en bloc resection highly challenging. Besides that, surgical resection poses risk of injury to the surrounding structures such as major blood vessels, bladder, ureters and rectum due to their proximity to the tumour. Therefore, multidisciplinary team approach is vital in ancipitating possible complications and preventing surgical morbidity. We present a case of advanced sacral chordoma which has been successfully resected with the assistance of pre-operative selective arterial embolization as a pre-emptive therapy. Case report: A 58-year-old lady presented with a large sacral chordoma $(17.17 \mathrm{~cm} \times 27.3$ $\mathrm{cm} \times 30.5 \mathrm{~cm}$ ) with sacral erosion, infiltration to gluteus maximus, medius and minimus muscles and lung metastasis. A decision to perform a surgical resection was made to alleviate the pain secondary to sacral nerve compression. Anticipating massive bleeding intra-operatively, pre-operative arterial embolization was performed involving one branch of right internal iliac artery as well as five branches of left internal and external iliac arteries using endovascular coils. The tumour was resected with partial sacrectomy from S2 and below. Intra-operatively, 6 pints of packed cells were transfused with estimated blood loss of 4 litres. The patient recovered well after the surgery. She was pain-free post-operatively with no lower limb neurological deficit. Conclusion: Surgical resection remains the treatment of choice for sacral chordoma. Pre-operative selective arterial embolization can reduce intra-operative bleeding and avoid a potentially convoluted surgery. 